\title{
Compound K Inhibits Autophagy-Mediated Apoptosis Through Activation of the PI3K- Akt Signaling Pathway Thus Protecting Against Ischemia/Reperfusion Injury
}

\author{
Xiangyan $\mathrm{Li}^{\mathrm{a}}$ Qingxia Huang ${ }^{\mathrm{b}}$ Manying Wang ${ }^{\mathrm{b}}$ Xiuci Yan ${ }^{\mathrm{b}, \mathrm{c}} \quad$ Xinying Song ${ }^{\mathrm{c}}$ \\ Rui Mac Rui Jiang ${ }^{c}$ Daqing Zhao ${ }^{a}$ Liwei Sun ${ }^{\mathrm{b}, c}$ \\ ajilin Provincial Key Laboratory of BioMacromolecules of Chinese Medicine, Jilin Ginseng Academy, \\ Changchun University of Chinese Medicine, Changchun, ${ }^{b}$ Research Center of Traditional Chinese \\ Medicine, the Affiliated Hospital to Changchun University of Chinese Medicine, Changchun, cJilin \\ Technology Innovation Center for Chinese Medicine Biotechnology, College of Biology and Chemistry, \\ Beihua University, Jilin, China
}

\section{Key Words}

Compound $\mathrm{K} \bullet$ Autophagy • Apoptosis • Ischemic/reperfusion injury • PI3K-Akt pathway

\begin{abstract}
Background/Aims: A series of reports revealed that autophagy and apoptosis exerted detrimental effects on the pathology of cardiac ischemia/reperfusion (I/R) injury. Ginsenoside compound $\mathrm{K}(\mathrm{CK})$, a major intestinal metabolite underlying the pharmacological actions of orally administered ginseng, has a protective effect against myocardial I/R injury. However, the molecular mechanisms by which CK protects against I/R injury remain unclear. In this study, we hypothesized that the cardioprotective effects of CK against I/R injury are mediated by inhibiting autophagy/apoptosis-related signaling pathways in H9c2 cardiomyocyte cells. Methods: $\mathrm{H} 9 \mathrm{c} 2$ cells were incubated with CK and exposed to I/R. Cell viability and damage was analyzed by MTT and lactate dehydrogenase assays. Reactive oxygen species (ROS) generation, mitochondrial damage, and cell apoptosis were analyzed by flow cytometry and TUNEL staining. The expression of autophagy, apoptosis, and related signaling proteins was analyzed by Western blotting and immunofluorescence staining. Results: CK pretreatment promoted cell viability and attenuated ROS accumulation and intracellular mitochondrial damage induced by I/R injury. Moreover, CK reduced autophagy by regulating the formation of phagocytic precursors to autophagosomes and also inhibited apoptosis through a mitochondrial-mediated pathway. Additionally, the cardioprotective effect of CK against I/R injury was mainly through the activation of the PI3K-Akt signaling pathway. Conclusions: CK pretreatment inhibits autophagy-mediated apoptosis induced by I/R injury through the

X. Li and Q. Huang contributed equally to this work.

\begin{tabular}{ll}
\hline Daqing Zhao & Jilin Prov. Key Lab. of BioMacromolecules of Chinese Med., Jilin Ginseng Academy \\
and Liwei Sun & Changchun Univ. of Chinese Med.,1035 Boshuo Road, Changchun, Jilin, 130021 (China) \\
& Tel. +86-431-86177630, E-Mail zhaodaqing1963@163.com, sunnylilwei@163.com
\end{tabular}
\end{abstract}




\section{Cellular Physiology Cell Physiol Biochem 2018:47:2589-2601 and Biochemistry Publisned online: July II, $2018 \quad \begin{aligned} & \text { Do 2018 The Authors. } \\ & \text { www.karger.com/cpb }\end{aligned}$ \\ Li et al.: Protective Effect of CK on Myocardial I/R Injury}

activation of the PI3K-Akt signaling pathway, which reveals that CK may be one of the key bioactive ingredients of ginseng for the treatment of myocardial I/R injury.

(C) 2018 The Author(s)

Published by S. Karger AG, Base

\section{Introduction}

Myocardial infarction is a major cause of death and disability worldwide [1]. Therapeutic strategies, including thrombolytic primary angioplasty and primary percutaneous coronary intervention, are frequently used to reduce the size of a myocardial infarct and improve the outcome in routine clinical practice [2]. Nevertheless, reperfusion results in a worsening of myocardial ischemia/reperfusion (I/R) injury, which may inevitably reduce the beneficial effects of reperfusion therapy [3]. Therefore, it is necessary to explore novel therapeutic strategies to reduce reperfusion-associated tissue injury after I/R.

Panax ginseng C.A. Meyer, as a traditional medicinal plant, has been used for over 2000 years to heal all aspects of the body [4]. Ginseng is thought to possess beneficial effects for the prevention and treatment of cardiovascular, endocrine, nervous system, and immune system diseases [5, 6]. At present, ginseng is commonly used in individuals who have cardiovascular risk factors, such as hypertension and hypercholesterolemia [7]. Moreover, ginseng and its ginsenoside constituents as bioactive ingredients are widely used in the clinical setting for treating cardiovascular diseases, especially myocardial I/R injury [8]. Recent studies have reported that the ginsenosides $\mathrm{Rb} 1$ and $\mathrm{Rb} 2$ have cardioprotective effects in models of myocardial I/R in vitro and in vivo [9]. Indeed, the ginsenosides $\mathrm{Rb} 1, \mathrm{Rb} 2, \mathrm{Rc}$, and $\mathrm{Rd}$ are transformed by intestinal bacteria to the major metabolite compound $\mathrm{K}$ (CK), that is, 20-0- $\beta$-(D-glucopyranosyl)-20(S)-protopanaxadiol (Fig. 1A), which is responsible for the pharmacological actions of orally administered ginseng [10]. It has been reported that CK has protective effects against I/R injury in a mouse model [11]. However, the mechanisms underlying the action of CK against I/R injury are unclear.

Autophagy is a cell survival mechanism that involves the degradation and recycling of cytoplasmic components [12]. If autophagic activity is insufficient, long-lived proteins and defective organelles accumulate to induce cell death [13]. In contrast, if autophagy destroys the cytosol and organelles beyond a certain threshold, autophagic cell apoptosis and death occur [14]. A variety of studies have suggested that autophagy during myocardial ischemia is cardiac protective and serves as a regenerative feedback mechanism, which suggests that enhancing autophagy could promote survival in response to mild forms of stress [15], but myocardial I/R-activated autophagy in the reperfusion period is detrimental and causes further myocardial damage [16]. The autophagy and apoptosis resulting from the reperfusion phase are accompanied by the activation of the PI3K-Akt signaling pathway and the up-regulation of Beclin-1 $[17,18]$. After the activation of Beclin-1, autophagy-related gene (Atg) proteins, including Atg5, Atg7, and Atg8 (microtubule-associated protein 1 light chain 3, LC3 in mammals), are involved in the nucleation and elongation of autophagosomes. Similarly, as one of the key autophagy substrates, sequestosome 1 (SQSTM1, p62) interacts directly with LC3 and brings p62-containing protein aggregates to the autophagosomes [19, $20]$. Thus, the prevention of excessive autophagy and apoptosis induced by I/R may reduce cardiomyocyte death and preserve cardiac function [21].

In this study, we aimed to investigate the protective effect of CK against I/R injury through the inhibition of autophagy and apoptosis via the PI3K-Akt pathway. To test this hypothesis, we used an autophagy inhibitor, 3-methyladenine (3-MA), and PI3K inhibitor, LY294002, to evaluate the potential mechanism of CK on autophagy and apoptosis in a model of I/R injury. Our results showed that autophagy-mediated apoptosis induced by I/R injury was significantly inhibited by CK through the activation of the PI3K-Akt signaling pathway. Our study reveals that CK may be one of the key bioactive ingredients of ginseng for the treatment of myocardial I/R injury. 


\section{Cellular Physiology \\ Cell Physiol Biochem 2018;47:2589-2601 \begin{tabular}{l|l} 
and Biochemistry Publisned onlne: July II, 2018 & $\begin{array}{l}\text { ○ 2018 The Author(s). Published by S. Karger AG, Basel } \\
\text { www.karger.com/cpb }\end{array}$ \\
\hline
\end{tabular} \\ Li et al.: Protective Effect of CK on Myocardial I/R Injury}

\section{Materials and Methods}

\section{Materials}

CK, 3-MA, and LY294002

(Sigma-Aldrich, St. Louis, MO,

USA) were dissolved in dimethyl sulfoxide (DMSO) and stored at $-20^{\circ} \mathrm{C}$ before use. Rabbit primary antibodies against mTOR (monoclonal, 1:1000, \#2983), phospho-mTOR (Ser2448, monoclonal, 1:1000, \#5536), phospho-Beclin-1 (monoclonal, 1:1000, Ser15, \#84966), Beclin-1 (monoclonal, 1:1000, \#3495), Cleaved Caspase-3 (polyclonal, 1:1000, \#9661), Bax (polyclonal, 1:1000, \#2772), LC3A/B (polyclonal, 1:1000,\#4108), Bcl2 (monoclonal, 1:1000, \#2870), SQSTM1/p62 (monoclonal, 1:1000, \#8025), phospho-Akt (1:1000, Ser473, \#4060), Akt (monoclonal, 1:1000, \#4691), Atg7 (monoclonal, 1:1000, \#8558), Atg5 (monoclonal, 1:1000, \#12994), PARP (monoclonal, 1:1000, \#9532), and mouse $\beta$-Actin (monoclonal, 1:2000, \#3700) were purchased from Cell Signaling Technology (Beverly, MA, USA).

$$
\begin{aligned}
& \text { Cell culture and } I / R \\
& \text { modeling }
\end{aligned}
$$

H9c2 cardiomyocyte cells were purchased from the American Type Culture Collection (Manassas, VA, USA). The cells were cultured in Dulbecco's modified Eagle's medium (DMEM; Gibco, New York, NY, USA) with high glucose supplemented with $10 \%$ fetal bovine serum (FBS; CLARK Bioscience, Claymont, DE, USA), $100 \mathrm{U} / \mathrm{mL}$ penicillin (Biosharp, Hefei, China), and $100 \mu \mathrm{g} / \mathrm{mL}$ streptomycin (Biosharp) and incubated at $37^{\circ} \mathrm{C}$ in a watersaturated atmosphere of $5 \%$ $\mathrm{CO}_{2}$. To establish the I/R injury model, H9c2 cells were seeded in a 96-well plate at a density of

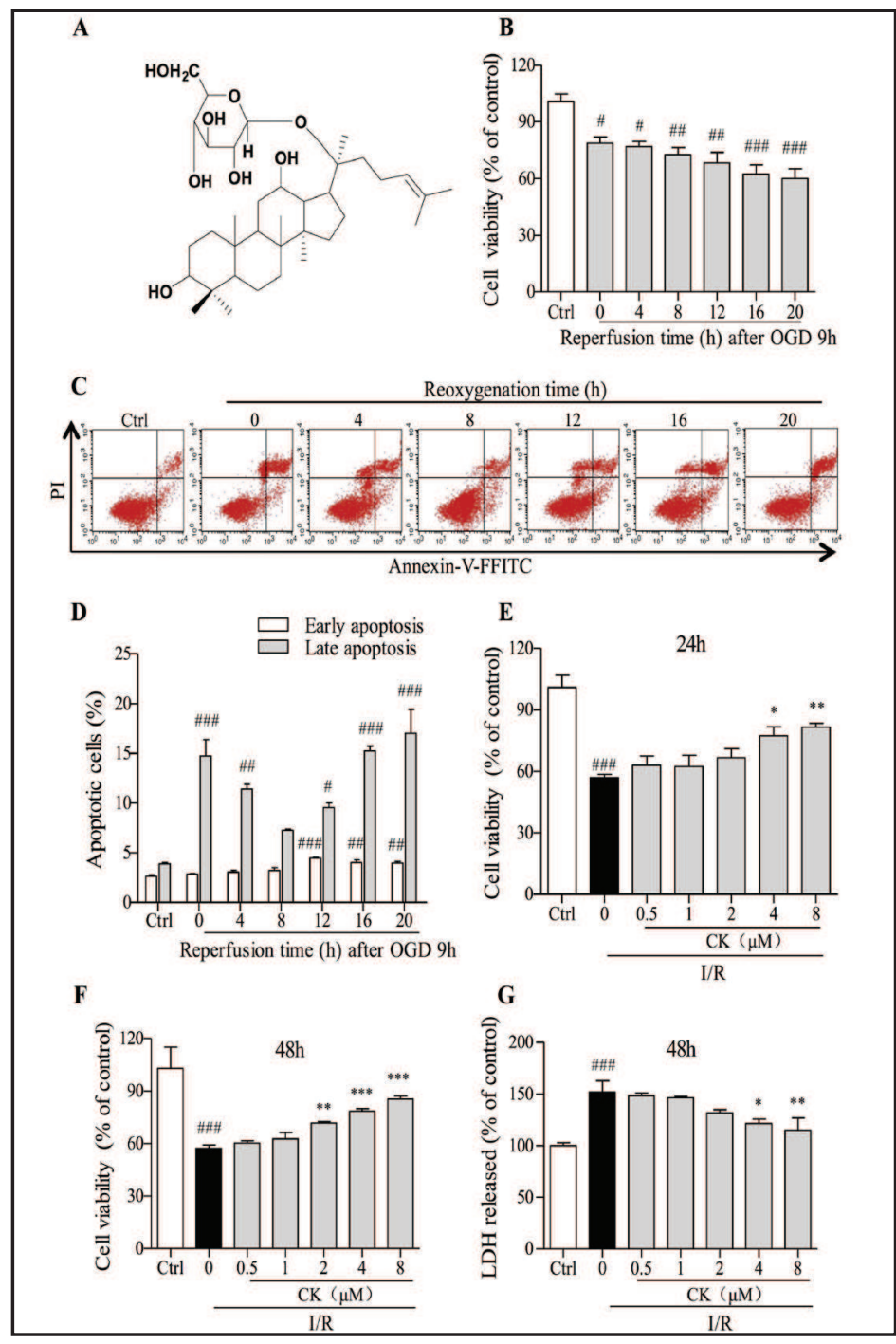

Fig. 1. CK pretreatment increases cell viability and inhibits cell damage induced by I/R. (A) Chemical structure of CK. (B) To investigate the protective effect of CK on I/R injury in H9c2 cardiomyocytes, the effect of reperfusion time after hypoxic incubation for $9 \mathrm{~h}$ on the viability of H9c2 cardiomyocytes was observed. (C) and (D) To investigate the protective effect of $\mathrm{CK}$ against I/R injury in H9c2 cardiomyocytes, the effect of reperfusion time on apoptosis after hypoxic incubation for 9 h was analyzed. (E) and (F) H9c2 cardiomyocyte viability following treatment with the indicated concentrations of CK for 24 and $48 \mathrm{~h}$ of I/R cell injury was assessed using an MTT assay. (G) Effect of CK for $48 \mathrm{~h}$ on the release of LDH in culture medium was determined at the end of reperfusion in $\mathrm{H} 9 \mathrm{c} 2$ cardiomyocytes was measured using an LDH assay kit. Ctrl: Control group; H9c2 cells were incubated in highglucose DMEM medium with $10 \%$ FBS under normoxia $\left(37^{\circ} \mathrm{C}, 5 \% \mathrm{CO}_{2}\right)$ in each experiment. ${ }^{\#} \mathrm{P}<0.05,{ }^{\# \#} \mathrm{P}<0.01,{ }^{\# \# \#} \mathrm{P}<0.001$ vs. the control group; ${ }^{*} \mathrm{P}<0.05,{ }^{* *} \mathrm{P}<0.01,{ }^{* * *} \mathrm{P}<0.001$ vs. the I/R group. 


\section{Cellular Physiology Cell Physiol Biochem 2018;47:2589-2601 and Biochemistry Published online: July II, $2018 \quad \begin{aligned} & \text { D } 2018 \text { The Authorst. } \\ & \text { www.karger.com/cpb }\end{aligned}$ \\ Li et al.: Protective Effect of CK on Myocardial I/R Injury}

$1.0 \times 10^{4}$ cells/well and grown for $24 \mathrm{~h}$. The medium was replaced with serum-free and glucose-free DMEM prior to culture under $95 \% \mathrm{~N}_{2}, 5 \% \mathrm{CO}_{2}$ by incubation for $3,6,9$, and $12 \mathrm{~h}$ (oxygen and glucose deprivation, OGD) in a BioSpa automated incubator (BioTek, Winooski, VT, USA). During the process of reperfusion, the cells were subjected to reperfusion by changing DMEM with high glucose containing $10 \%$ FBS followed by incubation under normoxia for $4,8,12,16$, and $20 \mathrm{~h} \mathrm{[22].}$

\section{MTT and lactate dehydrogenase (LDH) assays}

The viability of H9c2 cardiomyocytes treated with different concentrations of CK for 48 h was determined using an MTT assay. After treatment, cells cultured in 96-well plates were incubated with MTT solution $\left(0.5 \mathrm{mg} / \mathrm{mL}\right.$ final concentration) at $37^{\circ} \mathrm{C}$ for $4 \mathrm{~h}$. The formazan crystals were dissolved with DMSO, $100 \mu \mathrm{L} /$ well, and absorbance was detected at $570 \mathrm{~nm}$ on a microplate reader (Infinite M200 Pro, Tecan, Männedorf, Switzerland). The medium from H9c2 cardiomyocytes treated with different concentrations of CK under different conditions was collected to measure LDH release using an LDH assay kit (Jiancheng Bioengineering Institute, Nanjing, China) according to the manufacturer's instructions.

\section{Measurement of reactive oxygen species generation}

Cells treated with various concentrations of $\mathrm{CK}$ for $48 \mathrm{~h}$ prior to I/R injury were equilibrated in $10 \mu \mathrm{M}$ carboxy-H2DCFDA (Beyotime Biotechnology, Shanghai, China) in DMEM for 20 min at $37^{\circ} \mathrm{C}$ and washed three times with DMEM. Analysis was performed by flow cytometry on a FACS-Calibur (BD Biosciences, San Jose, CA, USA).

\section{Detection of mitochondrial membrane potential}

A JC-1 fluorescent probe (Beyotime Biotechnology) was used to estimate the effect of CK on mitochondrial membrane potential (MMP). The cells were cultured in 6 -well plates $\left(1.0 \times 10^{5}\right.$ cells/well $)$ and pretreated with various concentrations of CK for $48 \mathrm{~h}$ prior to I/R. After I/R, the cells were stained with JC-1 in the dark at $37^{\circ} \mathrm{C}$ for $20 \mathrm{~min}$ and observed immediately under a fluorescence microscopy or analyzed by a FACScan flow cytometer (BD Biosciences).

\section{Cell apoptosis by flow cytometry and TUNEL assay}

After treatment and I/R incubation in 6-well plates, cell apoptosis was analyzed using Annexin V-propidium iodide (PI) and TUNEL kits (Beyotime Biotechnology), according to the manufacturer's instruction. Briefly, the cells were harvested, washed twice with cold phosphate-buffered saline (PBS), and then incubated with $5 \mu \mathrm{L}$ Annexin V-FITC for $15 \mathrm{~min}$ and $10 \mu \mathrm{L}$ PI for $5 \mathrm{~min}$ in the dark at room temperature, respectively. Cellular fluorescence was measured with a flow cytometer (FACS Calibur ${ }^{\mathrm{TM}}$; BD Biosciences). For TUNEL staining, cells after treatment with CK and I/R incubation were rinsed with PBS and fixed with $4 \%$ paraformaldehyde for $40 \mathrm{~min}$. Then, the cells were permeabilized with $0.1 \%$ Triton X-100 for 10 min on ice and incubated in TUNEL reaction mixture for $60 \mathrm{~min}$ at $37^{\circ} \mathrm{C}$ in the dark. Finally, the cells were mounted with mounting medium, including DAPI (Beyotime Biotechnology), and covered with a glass coverslip. TUNEL-positive nuclei and the total number of nuclei were counted in at least 5 fields in three independent experiments.

\section{Immunofluorescence staining by confocal microscopy}

Briefly, H9c2 cells were placed in 12-well chamber slides (NEST Biotechnology Co., Ltd., Shanghai, China). After treatment with CK and I/R incubation, the cells were incubated with primary antibodies (1:200) and an Alexa Fluor 495-labeled goat anti-rabbit antibody (1:1000; BOSTER, Wuhan, China), and mounted with medium containing DAPI. A Nikon C2 confocal microscope with ZEN software (Nikon, Tokyo, Japan) was used to analyze the levels of p62, p-Akt, and $\beta$-Actin. Total laser intensity and photo multiplier gain were set constant for all groups and settings, and data were verified by two independent observers, who were blinded to the experimental group. A minimum of three coverslips were used for each experimental group, and at least three cell images were acquired from each coverslip.

Western blot analysis

Cell samples were lysed in RIPA buffer (Beyotime Biotechnology). Protein concentration was quantified with a BCA protein assay kit (Beyotime Biotechnology). Protein (30 $\mu \mathrm{g}$ ) was subjected to $10 \%$ or 


\section{Cellular Physiology Cell Physiol Biochem 2018;47:2589-2601

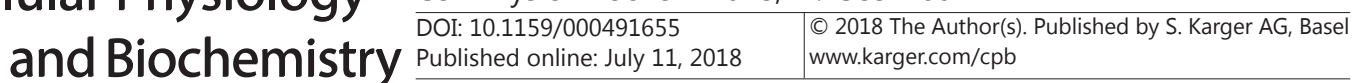 \\ Li et al.: Protective Effect of CK on Myocardial I/R Injury}

$12 \%$ sodium dodecyl sulfate polyacrylamide gel electrophoresis, transferred onto polyvinylidene fluoride membranes, and probed with primary antibodies overnight at $4^{\circ} \mathrm{C}$. $\beta$-Actin $(1: 1000)$ was used as a loading control. After incubation with appropriate secondary antibodies for $1 \mathrm{~h}$ at room temperature, protein bands were visualized using a chemiluminescent imaging system (FluorChem; ProteinSimple, San Jose, CA, USA).

\section{Statistical analysis}

Data from all experiments were analyzed using GraphPad Prism 6 (GraphPad Software, San Diego, CA). Data are presented as the mean \pm standard deviation for continuous variables. Student's two-tailed t test was used when there were only two groups for comparison, and one-way analysis of variance was performed to identify statistical significance in multiple-group comparisons. $P<$ 0.05 was considered statistically significant.

\section{Results}

CK promotes cell survival and reduces cell damage in $\mathrm{H} 9 \mathrm{c} 2$ cells after $I / R$ injury

To investigate the protective effect of $\mathrm{CK}$ on I/R injury in $\mathrm{H} 9 \mathrm{c} 2$ cardiomyocytes, the viability of $\mathrm{H} 9 \mathrm{c} 2$ cells incubated in hypoxia and no glucose/FBS (OGD) for different periods of time and followed by reperfusion for different periods of time was evaluated to establish a model of $\mathrm{I} / \mathrm{R}$ injury. Incubation under OGD for $9 \mathrm{~h}$ led to a $30 \%$ decrease in cell viability (Fig. 1B, $P$ $<0.05)$. In the model of I/R injury, reperfusion for 4,8 , 12,16 , and $20 \mathrm{~h}$ after $9 \mathrm{~h}$ OGD caused a decrease of $\mathrm{H} 9 \mathrm{c} 2$ cell viability in a timedependent manner, with approximately $60 \%$ viability after $20 \mathrm{~h}$ reperfusion (Fig. 1B, $P<0.001$ ). To confirm further reperfusion time after OGD, we examined the percentage of apoptotic cells at different times $(0$ to $20 \mathrm{~h}$ ). As shown in Fig. 1C and 1D, OGD for $9 \mathrm{~h}$

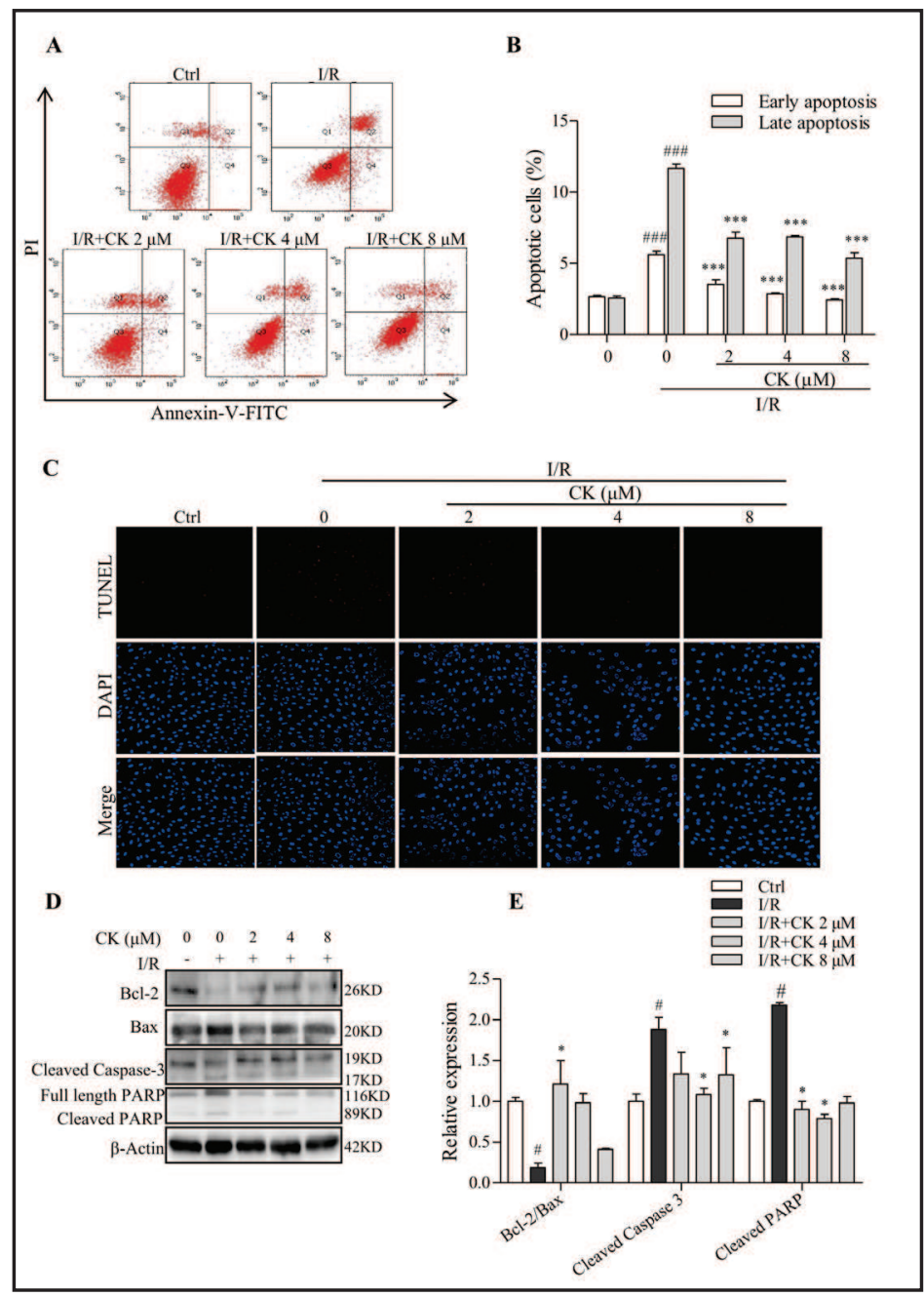

Fig. 2. CK protects $\mathrm{H} 9 \mathrm{c} 2$ cardiomyocytes against I/R-induced apoptosis. (A) After treatment with different concentrations of CK $(2,4$, or $8 \mu \mathrm{M})$ for $48 \mathrm{~h}, \mathrm{H} 9 \mathrm{c} 2$ cells were stained with Annexin V-FITC/PI to analyze the percentage of cells undergoing apoptosis by flow cytometry. (B) Bar diagram of early and late apoptotic cell rates from three separate experiments. (C) Representative images and bar graphs of TUNELpositive nuclei (red fluorescence). Blue: DAPI staining. (D) Expression levels of Bcl-2, Bax, Cleaved Caspase-3, and PARP were detected by Western blot analysis. $\beta$-Actin was used as a loading control. (E) Relative levels of Bcl-2, Bax, Cleaved Caspase-3, and cleaved PARP to $\beta$-Actin were expressed using bar graphs from three separate experiments. ${ }^{\#} \mathrm{P}<0.05,{ }^{\# \#} \mathrm{P}<0.01,{ }^{\# \# \#} \mathrm{P}<0.001$ vs. the control group; ${ }^{*} \mathrm{P}<0.05,{ }^{* * *} \mathrm{P}<0.001$ vs. the I/R group. 


\section{Cellular Physiology Cell Physiol Biochem 2018;47:2589-2601 \begin{tabular}{c|l}
\hline DOI: 10.1159/000491655 & @ 2018 The Author(s). Published by S. Karger AG, Basel
\end{tabular} \\ Li et al.: Protective Effect of CK on Myocardial I/R Injury}

caused late apoptosis (approximately 14.7\%). Reperfusion for 12, 16, and $20 \mathrm{~h}$ after OGD significantly increased both early apoptosis and late apoptosis $(P<0.01, P<0.001)$. On the basis of these findings, we confirmed the condition of I/R injury as $20 \mathrm{~h}$ of reperfusion after $9 \mathrm{~h}$ incubation with OGD for further study.

The potential cardioprotective effects of $\mathrm{CK}$ on H9c2 cardiomyocytes against I/R injury were estimated using MTT and LDH assays. Pretreatment with CK for $24 \mathrm{~h}(4$ or $8 \mu \mathrm{M})$ or $48 \mathrm{~h}(2,4$, or $8 \mu \mathrm{M})$ effectively ameliorated cell viability under $9 \mathrm{~h}$ incubation of OGD and subsequent reperfusion for $20 \mathrm{~h}$ in a concentration- and time-dependent manner (Fig. 1E and 1F; $P<0.05, P<0.01, P<0.001$ ). Moreover, LDH leakage, as a biomarker of cell death, was detected to evaluate the protective effect of CK on cell damage from I/R injury. As shown in Fig. 1G, CK treatment for $48 \mathrm{~h}$ significantly decreased the release of LDH induced by I/R injury $(P<0.05, P<0.01)$. These results indicated that CK pretreatment increased cell viability and decreased cell damage to protect against injury from OGD for $9 \mathrm{~h}$ and reperfusion for $20 \mathrm{~h}$.

CK decreases $I / R$ induced apoptosis in H9c2 cells

To observe the effect of $\mathrm{CK}$ on I/R-induced apoptosis, the cells were subjected to flow cytometry, TUNEL assay, and Western blot analysis. As shown in Fig. 2A and 2B, the early and late apoptosis induced by I/R injury was decreased after treatment with $\mathrm{CK}$ for $48 \mathrm{~h}(P<0.001)$. Moreover, the TUNEL assay showed that $\mathrm{CK}$ pretreatment reduced the percentage of TUNEL-positive cells caused by I/R injury, which was consistent with the analysis from flow cytometry (Fig. 2C). To analyze further the mechanism of $\mathrm{CK}$ against I/R-induced apoptosis, the expression levels of apoptosis-related proteins, such as Bcl-2, Bax, Cleaved Caspase-3, and PARP, were examined by Western blot analysis. The ratio of the antiapoptotic protein Bcl-2 and proapoptotic protein Bax was increased by CK pretreatment for 48 $h$ in $\mathrm{H} 9 \mathrm{c} 2$ cells with $\mathrm{I} / \mathrm{R}$ injury (Fig. 2D and 2E). Additionally, I/R injury

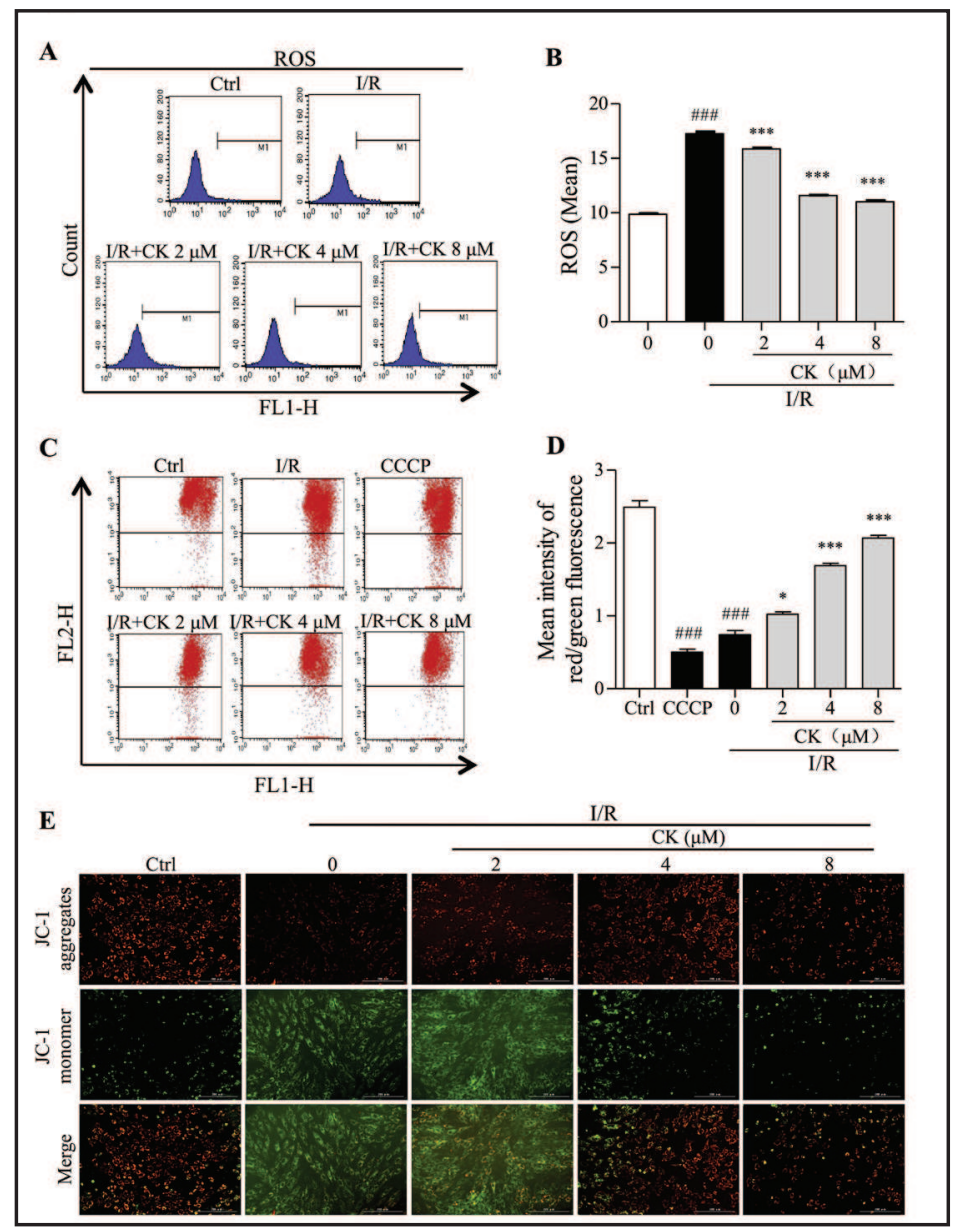

Fig. 3. $\mathrm{CK}$ pretreatment attenuates $\mathrm{I} / \mathrm{R}$-induced intracellular mitochondrial damage and ROS accumulation in H9c2 cardiomyocytes. (A) Intracellular ROS levels were evaluated by flow cytometry. (B) Bar diagram showing that CK decreased the I/R-induced accumulation of intracellular ROS from three separate experiments in H9c2 cardiomyocytes. (C) MMP was measured by flow cytometry. (D) Bar diagram showing that $\mathrm{CK}$ increased the ratio of red to green fluorescence intensity from three separate experiments in $\mathrm{H} 9 \mathrm{c} 2$ cardiomyocytes. CCCP: positive control. (E) Representative images of JC-1 red/green

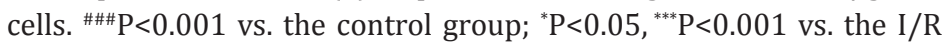
group. 


\section{Cellular Physiology Cell Physiol Biochem 2018;47:2589-2601 \begin{tabular}{c|l} 
DOI: 10.1159/000491655 & @ 2018 The Author(s). Published by S. Karger AG, Basel
\end{tabular} \\ Li et al.: Protective Effect of CK on Myocardial I/R Injury}

caused the Cleavage of Caspase- 3 and PARP in H9c2 cells, which was greatly decreased by CK treatment (Fig. 2D and 2E, $P<0.05$ ). Our results suggested that $\mathrm{CK}$ inhibited I/R-induced apoptosis in H9c2 cells through a mitochondrial-mediated apoptotic pathway.

\section{CK protects $\mathrm{H} 9 \mathrm{c} 2$ cardiomyocytes from intracellular reactive oxygen species accumulation} and mitochondrial membrane depolarization

Oxidative damage mediated by free radicals and mitochondrial damage also contributes to I/R-induced injury in cardiomyocytes. Compared with the control, I/R injury increased the level of intracellular reactive oxygen species (ROS) in H9c2 cells by approximately 1.75fold. CK preconditioning significantly inhibited the increase of ROS induced by I/R injury (Fig. 3A and 3B, $P<0.001$ ). MMP was assessed in I/R-induced H9c2 cells treated with CK using JC-1 staining and flow cytometry. I/R resulted in a pronounced decrease in the ratio of red to green fluorescence intensity (Fig. 3C, 3D, and $3 \mathrm{E}, P<0.001$ ), which is a sign of early stage apoptosis. These results indicated that CK preconditioning could protect cardiomyocytes against damage from intracellular ROS accumulation and mitochondrial membrane depolarization induced by I/R injury.

CK decreases autophagy in H9c2 cardiomyocytes following I/R injury

Autophagy is activated in the initial phase of hypoxia and is excessively enhanced in the reperfusion period. Our findings showed that CK pretreatment decreased ROS generation and increased Bcl-2 expression, which are strong promoters of autophagy through the up-regulation of Beclin-1. To determine whether CK treatment decreased autophagy in H9c2 cells, we observed the effect of CK on the expression of autophagy-related proteins using Western blot analysis and immunofluorescence staining. Beclin-1 is a platform protein that controls the initiation of autophagocytosis and distinct phases in endocytosis [23]. Our

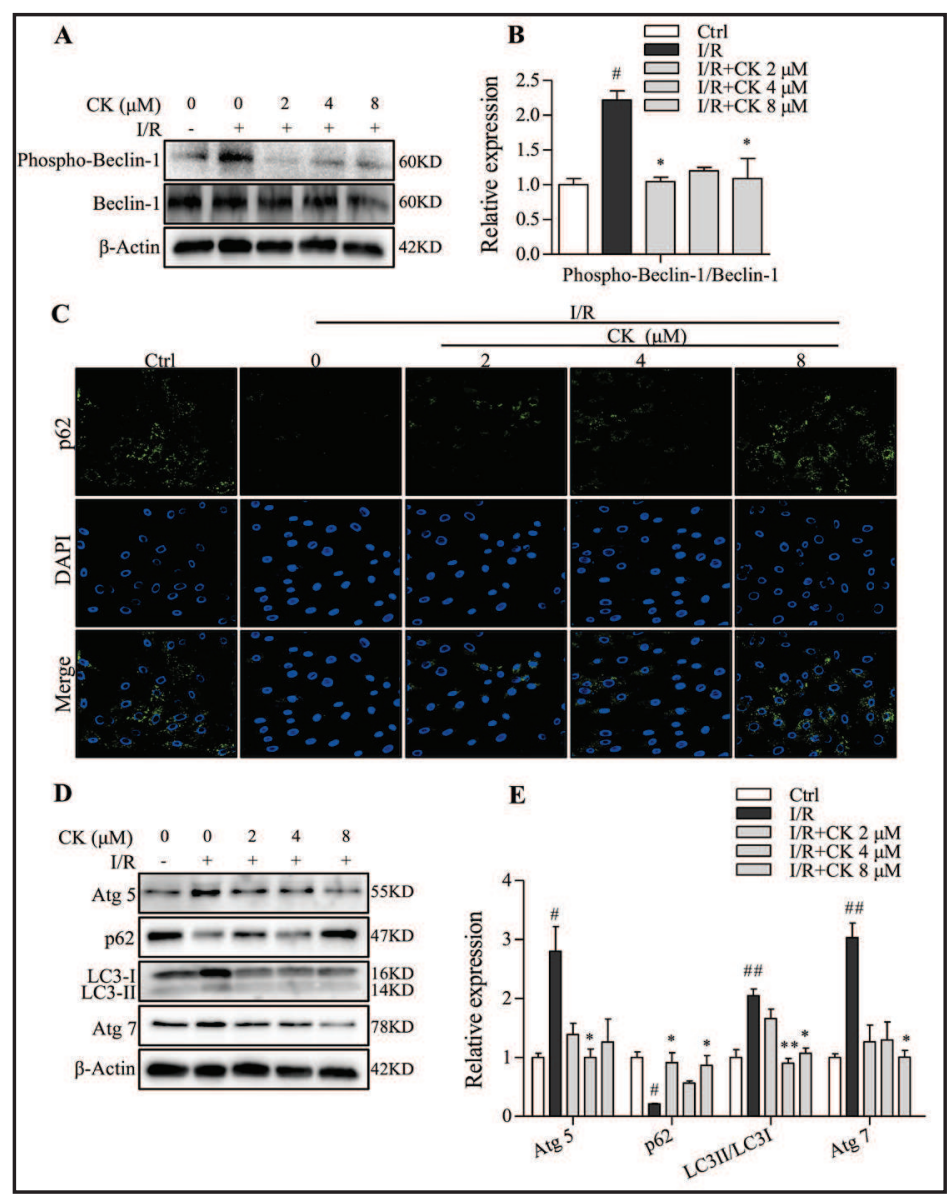

Fig. 4. CK decreases I/R-induced autophagy in H9c2 cardiomyocytes. (A) Expression levels of phospho-Beclin-1 and Beclin-1 in I/Rinjured $\mathrm{H} 9 \mathrm{c} 2$ cells treated with different concentrations of $\mathrm{CK}(2,4$, or $8 \mu \mathrm{M}$ ) for $48 \mathrm{~h}$ were detected by Western blot analysis. (B) Ratio of phospho-Beclin-1/Beclin-1 to $\beta$-Actin is shown in the bar graph. (C) Representative images of p62 staining in I/R-injured H9c2 cells treated with different concentrations of $\mathrm{CK}(2,4$, or $8 \mu \mathrm{M})$ for $48 \mathrm{~h}$. (D) Expression levels of Atg5, p62, LC3-II/I, and Atg7 were detected by immunoblotting analysis. (E) Relative intensity of protein levels to $\beta$-Actin was determined using densitometry analysis and shown in the bar graphs. ${ }^{\#} \mathrm{P}<0.05$, \#\# $\mathrm{P}<0.01$ vs. the control group; ${ }^{*} \mathrm{P}<0.05,{ }^{* *} \mathrm{P}<0.01$ vs. the I/R group. 


\section{Cellular Physiology \\ Cell Physiol Biochem 2018;47:2589-2601 and Biochemistry

results showed that the ratio of phospho-Beclin-1 to Beclin-1 was increased in $\mathrm{H} 9 \mathrm{c} 2$ cells exposed to I/R, which was greatly reduced by $\mathrm{CK}$ treatment (Fig. 4A and $4 \mathrm{~B}, P<0.05$ ). Moreover, p62 binds directly to LC3 via a specific sequence motif and is itself degraded by autophagy to facilitate the degradation of protein aggregates [24]. Staining for $\mathrm{p} 62$ showed that I/R injury decreased the expression of p62 in $\mathrm{H} 9 \mathrm{c} 2$ cells, which was reversed by CK pretreatment (Fig. 4C). In addition, Atg5 recruits phagocytic precursors to form phagophores, while the related Atg proteins, such as p62, LC3II/ LC3I (Atg8), and Atg7, regulate phagophore-toautophagosome formation [25]. We found that the expression of Atg5, LC3II/I, and Atg7 was increased in H9c2 cells exposed to

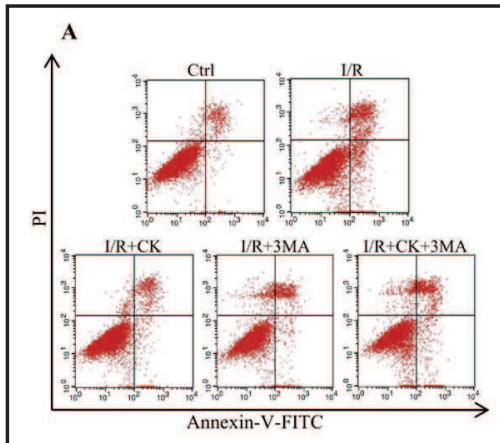

C

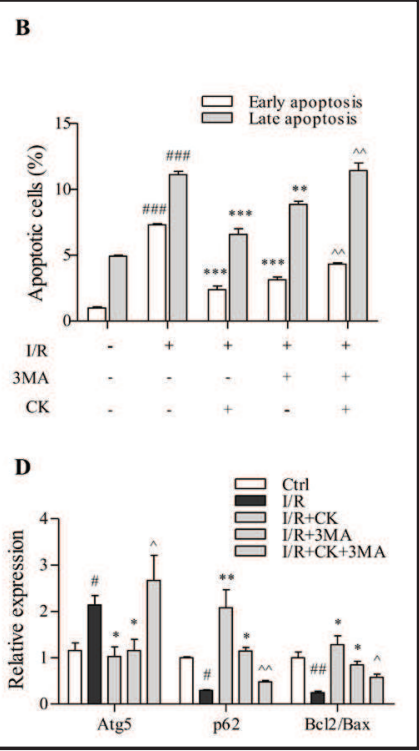

Fig. 5. CK protects cardiomyocytes by inhibiting autophagy-mediated apoptosis in $\mathrm{H} 9 \mathrm{c} 2$ cells subjected to I/R. (A) H9c2 cells were treated with CK for $48 \mathrm{~h}, 3-\mathrm{MA}$, or their combination for $12 \mathrm{~h}$, followed by I/R injury and harvested for Annexin V-FITC/PI staining to analyze apoptosis by flow cytometry. (B) Bar diagram of apoptotic cell rates (early and late apoptosis) from A in three separate experiments. (C) Expression levels of Atg5, p62, Bcl-2, and Bax were detected by Western blot analysis. (D) Relative intensity of protein levels (Atg5, p62, Bcl-2, and Bax) was determined using densitometric analysis. ${ }^{\#} \mathrm{P}<0.05$, ${ }^{\# \# \#} \mathrm{P}<0.001$ vs. the control group; ${ }^{*} \mathrm{P}<0.05,{ }^{* *} \mathrm{P}<0.01,{ }^{* * *} \mathrm{P}<0.001$ vs. the I/R group; ${ }^{\wedge} \mathrm{P}<0.05$, ${ }^{\wedge \wedge} \mathrm{P}<0.01$ vs. the $\mathrm{I} / \mathrm{R}+\mathrm{CK}$ group. $I / R$, which was greatly attenuated by different concentrations of CK (Fig. 4D and 4E, $P<0.05, P<0.01$ ). As expected, Western blot analysis showed that CK treatment increased the expression of p62 in H9c2 cells exposed to I/R (Fig. 4D and 4E, $P<0.05$ ), which was similar to the finding shown in Fig. 4C. Taken together, these results suggest that CK reduced the autophagy induced by I/R injury by regulating the formation of autophagosomes by phagocytic precursors in $\mathrm{H} 9 \mathrm{c} 2$ cells.

Autophagy-induced apoptosis is involved in the cardioprotective effects of CK in H9c2 cells

To further confirm our hypothesis that the inhibition of autophagy-induced apoptosis could be one of the mechanisms underlying the effect of CK on the recovery from I/R injury, we used a classical autophagy inhibitor, 3-MA, which interferes with the formation of autophagosomes in mammalian cells by inhibiting class III PI3K activity to clarify the relationship between apoptosis and autophagy inhibited by CK. As shown in Fig. 5A and $5 B$, the percentage of apoptotic cells induced by I/R was suppressed by $C K$ or 3-MA alone treatment. The combination of 3-MA and CK significantly increased the percentage of cells undergoing early and late apoptosis in the I/R environment compared with $\mathrm{CK}$ alone, which was similar with the I/R group $(P<0.01$ or $P<0.001)$. To verify further the effect of CK on alleviating apoptosis induced by I/R through autophagy-mediated pathways, we detected the expression of key autophagy and apoptosis proteins, such as Atg5, p62, Bcl-2, and Bax. Compared with the I/R+CK group, the combination of 3-MA with CK in the I/R group had no obvious effect on decreasing the expression of Atg5 and increasing the expression of p62. In addition, the ratio of $\mathrm{Bcl}-2$ to Bax in the group with the combination of 3-MA and CK was lower than that in the I/R+CK group (Fig. 5C and 5D, $P<0.05$ or $P<0.01$ ). Overall, the results 


\section{Cellular Physiology \\ Cell Physiol Biochem 2018;47:2589-2601 and Biochemistry

demonstrated that the antiapoptosis effect of CK in the $I / R$ environment was attenuated by 3-MA, suggesting that $\mathrm{CK}$ alleviated apoptosis induced by $I / R$ through a u t o phagy-mediated pathways.

CK inhibits autophagy and apoptosis through the activation of the PI3K-Akt pathway in $H 9 c 2$ cells subjected to $I / R$

The

stimulatory signaling axis of PI3K-Akt-mTOR pathways usually represses both autophagy and apoptosis, meaning that the activation of the PI3KAkt pathway may serve as a potential mechanism for the treatment of cardiac I/R injury [26]. Moreover, AKT can phosphorylate mTOR to inhibit its pro-autophagic and pro-apoptotic functions, respectively [27]. To explore the molecular mechanisms by which CK protects against I/R injury, we used a PI3K inhibitor, LY294002, to evaluate the potential protective role of CK in autophagy-mediated apoptosis induced by I/R through the activation of PI3K signaling. As shown in Fig. 6A and 6B, the percentage of cells undergoing early and late apoptosis induced by I/R was suppressed by CK treatment $(P<0.001)$, which was attenuated by the combination of CK with LY294002 $(P<0.05$ or $P<0.01)$. The ratio of Bcl-2 to Bax and p62 was higher in the I/R+CK group than with the combination of LY294002 and CK by Western blot analysis. In addition, CK treatment led to significant decreases in the expression of apoptosis markers, PARP cleavage, and autophagy markers, LC3II/I, compared to the I/R group, which were blocked by LY294002 (Fig. 6C and 6D, $P<0.05$ or $P<0.01$ ).

To further confirm the effect of CK treatment on the PI3K-Akt pathway, we assessed related proteins from the PI3K-Akt pathway, namely, p-Akt (Ser473) and p-mTOR (Ser2448), by Western blot analysis in $\mathrm{H} 9 \mathrm{c} 2$ cells. CK treatment resulted in a significant increase in the phosphorylation of Akt and mTOR compared with the I/R group, which was blocked 


\section{Cellular Physiology Cell Physiol Biochem 2018;47:2589-2601 and Biochemistry Publisned online: July II, $2018 \quad \begin{aligned} & \text { D 2018 The Authors. } \\ & \text { www.karger.com/cpb }\end{aligned}$ \\ Li et al.: Protective Effect of CK on Myocardial I/R Injury}

by LY294002 (Fig. 6E, $P<0.05$ or $P<0.01$ ). Immunofluorescence staining showed that $\mathrm{I} / \mathrm{R}$ decreased the level of p-Akt (Ser473) in H9c2 cells, which was reversed by CK treatment. The combination of CK with LY294002 did not obviously increase the level of p-Akt in the I/R cell model compared to CK treatment (Fig. 6F). These data provide evidence that CK treatment attenuated autophagy and apoptosis through the activation of the PI3K-Akt pathway during I/R injury.

\section{Discussion}

Initial therapy for acute myocardial infarction is directed toward the restoration of blood supply as soon as possible to reduce damage to the myocardium [28]. However, reperfusion injury remains a major clinical problem that has failed to be improved by multiple pharmacologic approaches [29]. The inhibition of autophagy and apoptosis during the stress of I/R could provide a therapeutic target for cardiac protection. In the present study, we used a cardiomyocyte I/R model to demonstrate that $\mathrm{CK}$, a key metabolite from ginseng after oral administration, exerts a protective effect against I/R-induced injury. Our findings identify a novel mechanism underlying the action of CK on the inhibition of autophagy and apoptosis and provide insights for the therapeutic use of ginseng for the treatment of cardiac disease.

After ischemia, a long period of secondary myocardial injury occurs following myocardial reperfusion, including oxidative stress, inflammation, apoptosis, and autophagy. During reperfusion, the reentry of oxygenated blood into ischemic tissue facilitates ATP restoration and results in the generation of ROS to exacerbate ischemic injury [30]. Being inhibited by low $\mathrm{pH}$, the mitochondrial permeability transition pore (mPTP) is kept quiescent during ischemia [31]. Upon reperfusion, the huge increase of ROS induces the opening of the MPTP, which leads to cell and tissue damage [32]. Thus, antioxidant therapy could be effective in preventing oxidative stress-induced cell injury during I/R. We found that I/R-induced oxidative stress, MMP depolarization, and cell damage were effectively blocked by CK preconditioning in a dose-dependent manner.

Recent studies revealed that autophagy exerted detrimental effects in the pathology of cardiac I/R injury [33]. The process of autophagosome formation involves two major steps: nucleation and elongation of the isolation membrane. The UNC-51-like kinase/ Atg1 kinase complex, class III PI3K complex, and their related proteins are important for the nucleation step, whereas the Atg12- and LC3/Atg8-conjugation systems are crucial for the elongation step [34]. Beclin-1 is required for autophagy vesicle nucleation, which is dramatically enhanced after I/R in the area at risk of myocardial infarction [35]. Moreover, LC3 plays a crucial role during the ensuing autophagosome elongation step, which serves as a widely used marker for autophagosomes [36]. Additionally, p62 is purported to act as an adaptor molecule linking ubiquitinated proteins to the autophagic machinery and interActing with LC3-II in the detection of different stages of autophagic vesicles [37]. In the present study, the phosphorylation of Beclin-1 and the ratio of LC3-II/LC3-I were increased after I/R injury, which were reversed significantly by CK. A specific inhibitor of autophagy, 3-MA, was used to explore whether CK played a protective role against I/R injury through autophagy. We found that the effect of CK on autophagy induced by I/R injury was abolished by 3-MA, which confirms that autophagy is a primary event during the secondary damage following reperfusion. To our knowledge, this is the first study indicating that CK protects cardiomyocytes against I/R injury through the inhibition of excessive autophagy.

The interplay between autophagy and apoptosis is highlighted by the fact that a regulator of apoptosis, namely, the Bcl-2 family, regulates autophagy. Conversely, certain autophagic proteins, such as Atg5 and Beclin-1, play important roles in apoptosis [38]. In myocardial I/R injury, autophagy may also promote cell death through the excessive self-digestion and degradation of essential cellular constituents or interaction with apoptotic cascade [39], which could be considered a potential target to decrease apoptosis. We chose several autophagy/ apoptosis markers, such as Atg5, p62, and the Bcl-2/Bax ratio, using multiple assays to 


\section{Cellular Physiology \\ Cell Physiol Biochem 2018;47:2589-2601 and Biochemistry

assess better the effect of CK on autophagy-mediated apoptosis. Several lines of evidence demonstrated that the anti-apoptotic activity of CK through inhibiting autophagy was dramatically abolished by treatment with 3-MA.

The PI3K-Akt pathway, the main downstream signal activated by $\mathrm{I} / \mathrm{R}$ injury, is particularly important for mediating myocardial survival under a wide variety of circumstances [20]. Akt phosphorylation can activate mTOR to inhibit autophagy and apoptosis during I/R injury [40]. It has been reported that the PI3K-Akt pathway partially mediates CK-mediated survival in a rat model of myocardial I/R injury [11]. Similarly, our data showed that the cardioprotective effect of CK was blocked by LY294002, suggesting that PI3K-Akt activation underlies the mechanism of CK on the inhibition of autophagy-mediated cell

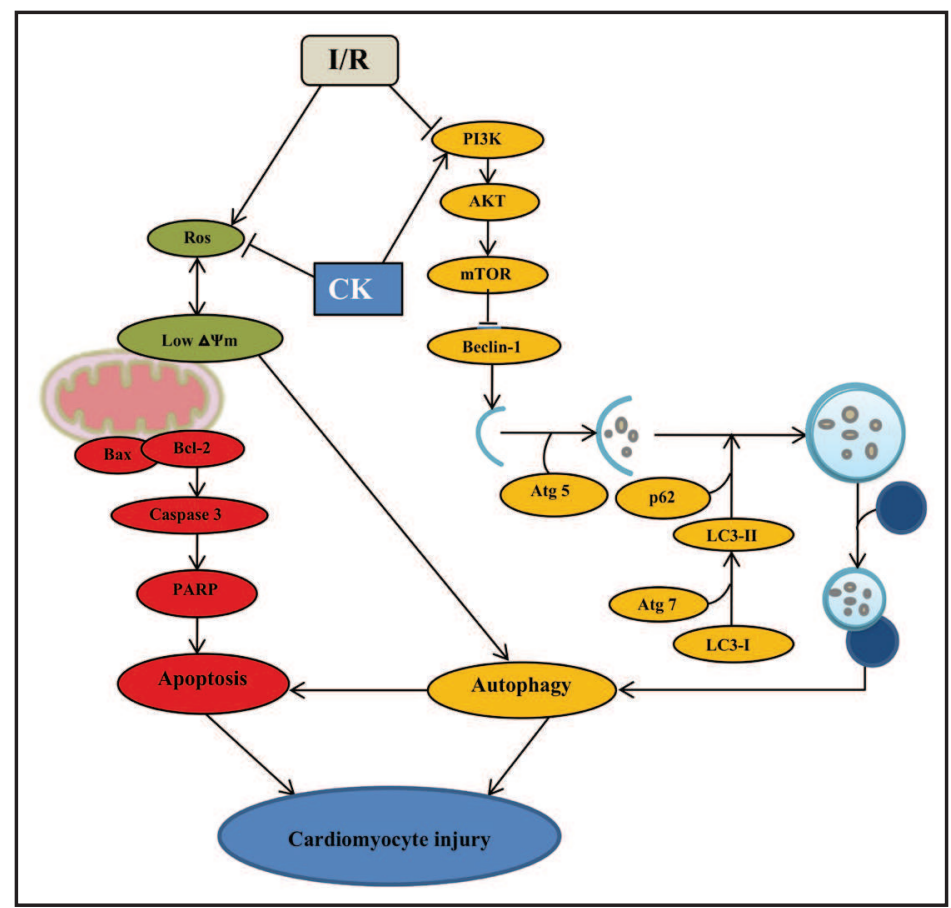

Fig. 7. Schematic illustration of the mechanisms underlying $\mathrm{CK}$ protection from I/R-induced autophagy and apoptosis in H9c2 cells. CK preconditioning significantly inhibited ROS generation and MMP induced by I/R injury. CK pretreatment reduced autophagy induced by $\mathrm{I} / \mathrm{R}$ by regulating the formation of phagocytic precursors to autophagosomes. CK also inhibited I/R-induced apoptosis by reducing the levels of apoptosis-related proteins such as Bcl-2/Bax and increasing the cleavage of Caspase- 3 and PARP, which was mediated by autophagy. Importantly, the protective effect of CK against autophagy and apoptosis induced by I/R was mainly through the activation of the PI3K-Akt signaling pathway. death against I/R injury.

\section{Conclusion}

In summary, we, for the first time, have demonstrated that CK provides a superior cardioprotective effect against I/R injury by reducing ROS, restoring MMP, and inhibiting myocardial autophagy-mediated apoptosis in H9c2 cardiomyocytes. Furthermore, our results illustrated that the cardioprotective effect of CK against I/R injury is mainly through the activation of the PI3K-Akt signaling pathway (Fig. 7). Our findings suggest that CK may be suitable for recovery from myocardial I/R injury as a key bioactive ingredient of ginseng.

\section{Acknowledgments}

This work was supported by Advanced Technological Innovation Platform of Jilin Province Higher Education for Ginseng, National Natural Science Foundation of China (No. 81641138, No. 81602257), The National Key Research and Development Program of China(2017YFC1702103),Science and Technology Project of Jilin Province, China (No. 201677), Science and Technology Development Plan of Jilin Province, China (No. 20160307027YY), Development and Reform Commission Project of Jilin Province, China (No. 2017C043). 


\section{Cellular Physiology Cell Physiol Biochem 2018;47:2589-2601 \begin{tabular}{l|l|l}
$: 10.1159 / 000491655$ & () 2018 The Author(s). Published by S. Karger AG, Basel
\end{tabular} and Biochemistry Publisned onIne: July II, 2018 www.karger.com/cpb}

Li et al.: Protective Effect of CK on Myocardial I/R Injury

\section{Disclosure Statement}

The authors declare to have no competing interests.

\section{References}

1 Hausenloy DJ, Yellon DM: Myocardial ischemia-reperfusion injury: a neglected therapeutic target. J Clin Invest 2013;123:92-100.

-2 Silber S: Secondary prevention after myocardial infarction with prolonged dual anti-platelet therapy (DAPT). A new therapeutic concept. MMW Fortschr Med 2017;159:49-52.

3 Kalogeris T, Baines CP, Krenz M, Korthuis RJ: Cell biology of ischemia/reperfusion injury. Int Rev Cell Mol Biol 2012;298:229-317.

4 Chen X, Wang M, Xu X, Liu J, Mei B, Fu P, Zhao D, Sun L: Panax ginseng total protein promotes proliferation and secretion of collagen in NIH/3T3 cells by activating extracellular signal-related kinase pathway. J Ginseng Res 2017;41:411-418.

5 Joh EH, Lee IA, Jung IH, Kim DH: Ginsenoside Rb1 and its metabolite compound K inhibit IRAK-1 activation-the key step of inflammation. Biochem Pharmacol 2011;82:278-286.

6 Park JS, Shin JA, Jung JS, Hyun JW, Van Le TK, Kim DH, Park EM, Kim HS: Anti-inflammatory mechanism of compound $\mathrm{K}$ in activated microglia and its neuroprotective effect on experimental stroke in mice. J Pharmacol Exp Ther 2012;341:59-67.

7 Kim JH: Cardiovascular Diseases and Panax ginseng: A Review on Molecular Mechanisms and Medical Applications. J Ginseng Res 2012;36:16-26.

8 Li F, Zhang Y, Zeng D, Xia Y, Fan X, Tan Y, Kou J, Yu B: The Combination of Three Components Derived from Sheng MaiSan Protects Myocardial Ischemic Diseases and Inhibits Oxidative Stress via Modulating MAPKs and JAK2-STAT3 Signaling Pathways Based on Bioinformatics Approach. Front Pharmacol 2017;8:21.

-9 Yan X, Liu J, Wu H, Liu Y, Zheng S, Zhang C, Yang C: Impact of miR-208 and its Target Gene Nemo-Like Kinase on the Protective Effect of Ginsenoside Rb1 in Hypoxia/Ischemia Injuried Cardiomyocytes. Cell Physiol Biochem 2016;39:1187-1195.

10 Wang CZ, Du GJ, Zhang Z, Wen XD, Calway T, Zhen Z, Musch MW, Bissonnette M, Chang EB, Yuan CS: Ginsenoside compound $\mathrm{K}$, not $\mathrm{Rb} 1$, possesses potential chemopreventive activities in human colorectal cancer. Int J Oncol 2012;40:1970-1976.

11 Tsutsumi YM, Tsutsumi R, Mawatari K, Nakaya Y, Kinoshita M, Tanaka K, Oshita S: Compound K, a metabolite of ginsenosides, induces cardiac protection mediated nitric oxide via Akt/PI3K pathway. Life Sci 2011;88:725-729.

12 Apel A, Zentgraf H, Buchler MW, Herr I: Autophagy-A double-edged sword in oncology. Int J Cancer 2009;125:991-995.

13 Decuypere JP, Parys JB, Bultynck G: Regulation of the autophagic bcl-2/beclin 1 interaction. Cells 2012;1:284-312.

14 Huang Z, Han Z, Ye B, Dai Z, Shan P, Lu Z, Dai K, Wang C, Huang W: Berberine alleviates cardiac ischemia/ reperfusion injury by inhibiting excessive autophagy in cardiomyocytes. Eur J Pharmacol 2015;762:1-10.

15 Li Q Dong QT, Yang YJ, Tian XQ Jin C, Huang PS, Jiang LP, Chen GH: AMPK-mediated cardioprotection of atorvastatin relates to the reduction of apoptosis and activation of autophagy in infarcted rat hearts. Am J Transl Res 2016;8:4160-4171.

16 Xiao J, Zhu X, Kang B, Xu J, Wu L, Hong J, Zhang Y, Ni X, Wang Z: Hydrogen Sulfide Attenuates Myocardial Hypoxia-Reoxygenation Injury by Inhibiting Autophagy via mTOR Activation. Cell Physiol Biochem 2015;37:2444.

17 Ma X, Liu H, Foyil SR, Godar RJ, Weinheimer CJ, Diwan A: Autophagy is impaired in cardiac ischemiareperfusion injury. Autophagy 2012;8:1394-1396.

18 Ghavami S, Gupta S, Ambrose E, Hnatowich M, Freed DH, Dixon IM: Autophagy and heart disease: implications for cardiac ischemia-reperfusion damage. Curr Mol Med 2014;14:616-629.

19 Chen C, Hu LX, Dong T, Wang GQ, Wang LH, Zhou XP, Jiang Y, Murao K, Lu SQ Chen JW, Zhang GX: Apoptosis and autophagy contribute to gender difference in cardiac ischemia-reperfusion induced injury in rats. Life Sci 2013;93:265-270. 


\section{Cellular Physiology Cell Physiol Biochem 2018;47:2589-2601 \begin{tabular}{l|l|l}
\cline { 2 - 4 } DOI: 10.1159/000491655 & () 2018 The Author(s). Published by S. Karger AG, Basel
\end{tabular} and Biochemistry Published onmine: July II, 2018 www.karger.com/cpb}

Li et al.: Protective Effect of CK on Myocardial I/R Injury

20 Ma X, Liu H, Foyil SR, Godar RJ, Weinheimer CJ, Hill JA, Diwan A: Impaired autophagosome clearance contributes to cardiomyocyte death in ischemia/reperfusion injury. Circulation 2012;125:3170-3181.

-21 Wang F, Jia J, Rodrigues B: Autophagy, Metabolic Disease, and Pathogenesis of Heart Dysfunction. Can J Cardiol 2017;33:850-859.

22 Su F, Myers VD, Knezevic T, Wang J, Gao E, Madesh M, Tahrir FG, Gupta MK, Gordon J, Rabinowitz J, Ramsey FV, Tilley DG, Khalili K, Cheung JY, Feldman AM: Bcl-2-associated athanogene 3 protects the heart from ischemia/reperfusion injury. JCI Insight 2016;1:e90931.

-23 Salminen A, Kaarniranta K, Kauppinen A: Beclin 1 interactome controls the crosstalk between apoptosis, autophagy and inflammasome activation: impact on the aging process. Ageing Res Rev 2013;12:520-534.

24 Schlafli AM, Berezowska S, Adams O, Langer R, Tschan MP: Reliable LC3 and p62 autophagy marker detection in formalin fixed paraffin embedded human tissue by immunohistochemistry. Eur J Histochem 2015;59:2481.

-25 Marino G, Niso-Santano M, Baehrecke EH, Kroemer G: Self-consumption: the interplay of autophagy and apoptosis. Nat Rev Mol Cell Biol 2014;15:81-94.

26 Wang Z, Wang Y, Ye J, Lu X, Cheng Y, Xiang L, Chen L, Feng W, Shi H, Yu X, Lin L, Zhang H, Xiao J, Li X: bFGF attenuates endoplasmic reticulum stress and mitochondrial injury on myocardial ischaemia/reperfusion via activation of PI3K/Akt/ERK1/2 pathway. J Cell Mol Med 2015;19:595-607.

27 Yoshida GJ: Therapeutic strategies of drug repositioning targeting autophagy to induce cancer cell death: from pathophysiology to treatment. J Hematol Oncol 2017;10:67.

28 Wang M, Vaez M, Dorner TE, Tiihonen J, Voss M, Ivert T, Mittendorfer-Rutz E: Trajectories and characteristics of work disability before and after acute myocardial infarction. Heart 2017;10.1136/ heartjnl-2017-311950

29 Han JY, Li Q Ma ZZ, Fan JY: Effects and mechanisms of compound Chinese medicine and major ingredients on microcirculatory dysfunction and organ injury induced by ischemia/reperfusion. Pharmacol Ther 2017;177:146-173.

- 30 Nishida K, Yamaguchi 0, Otsu K: Crosstalk between autophagy and apoptosis in heart disease. Circ Res 2008;103:343-351.

-31 Webster KA: Mitochondrial membrane permeabilization and cell death during myocardial infarction: roles of calcium and reactive oxygen species. Future Cardiol 2012;8:863-884.

-32 Motloch LJ, Hu J, Akar FG: The mitochondrial translocator protein and arrhythmogenesis in ischemic heart disease. Oxid Med Cell Longev 2015;2015:234104.

33 Ma S, Wang Y, Chen Y, Cao F: The role of the autophagy in myocardial ischemia/reperfusion injury. Biochim Biophys Acta 2015;1852:271-276.

34 Mizushima N, Yoshimori T, Levine B: Methods in mammalian autophagy research. Cell 2010;140:313-326.

-35 Matsui Y, Takagi H, Qu X, Abdellatif M, Sakoda H, Asano T, Levine B, Sadoshima J: Distinct roles of autophagy in the heart during ischemia and reperfusion: roles of AMP-activated protein kinase and Beclin 1 in mediating autophagy. Circ Res 2007;100:914-922.

36 Mizushima N, Yamamoto A, Matsui M, Yoshimori T, Ohsumi Y: In vivo analysis of autophagy in response to nutrient starvation using transgenic mice expressing a fluorescent autophagosome marker. Mol Biol Cell 2004;15:1101-1111.

-37 Jiang T, Harder B, Rojo de la Vega M, Wong PK, Chapman E, Zhang DD: p62 links autophagy and Nrf2 signaling. Free Radic Biol Med 2015;88:199-204.

38 Schiattarella GG, Hill JA: Therapeutic targeting of autophagy in cardiovascular disease. J Mol Cell Cardiol 2016;95:86-93.

-39 Hamacher-Brady A, Brady NR, Logue SE, Sayen MR, Jinno M, Kirshenbaum LA, Gottlieb RA, Gustafsson AB: Response to myocardial ischemia/reperfusion injury involves Bnip3 and autophagy. Cell Death Differ 2007;14:146-157.

40 Ebrahimi S, Hosseini M, Shahidsales S, Maftouh M, Ferns GA, Ghayour-Mobarhan M, Hassanian SM, Avan A: Targeting the Akt/PI3K Signaling Pathway as a Potential Therapeutic Strategy for the Treatment of Pancreatic Cancer. Curr Med Chem 2017;24:1321-1331. 